\title{
How does the estimated phase of menstrual cycle or menopause influence the prevalence of vasovagal syncope induced by head-up tilt test
}

\author{
Artur Z. Pietrucha, Joanna Jędrzejczyk-Spaho, Ewa Konduracka, Irena Bzukała, \\ Krzysztof Krawczyk, Olga Kruszelnicka-Kwiatkowska, Jadwiga Nessler \\ Syncope Unit, Department of Coronary Artery Disease and Heart Failure, Institute of Cardiology, \\ Medical School of Jagiellonian University, John Paul II Hospital, Krakow, Poland
}

\begin{abstract}
Background: The purpose of this study was to evaluate the prevalence of syncope induced by head-up tilt test (HUTT) and the type of positive vasovagal response to the orthostatic stress in a relationship to the estimated phase of menstrual cycle (follicular phase, luteal phase) or menopause.

Methods: We observed a cohort of 500 women at age 13-89 years (median of age 37.5), referred to HUTT. Phase of the menstrual cycle was determined on the basis of the usual length of menstrual cycle and the day of the cycle at the time of the study. We assumed that the length of the luteal phase is constant and it is 14 days.

Results: In premenopausal patients, the occurrence of the mixed and cardioinhibitory response was significantly more frequent in comparison to postmenopausal women $(48.8 \mathrm{vs} .35 .1 \%$ and $19.7 \mathrm{vs} .12 .4 \%$, respectively; $p<0.00001)$, while the occurrence of the vasodepressive one was significantly less frequent $(7.3 \%$ vs. $28.6 \% ; p<0.00001)$ in that group of patients. Women in follicular phase presented vasodepressive response during HUTT more often than woman in the luteal phase (10.0\% vs. $4.1 \%, p<0.00001)$. Conclusions: Among women referred for HUTT, the prevalence of the vasovagal syndrome did not differ between those that were pre- and post-menopausal. Higher incidence of vasodepressive reaction in postmenopausal women was observed. Among the premenopausal women, the vasodepressive type of vasovagal syndrome occurred more often in follicular then in luteal phase. (Cardiol J 2017; 24, 5: 523-529)

Key words: head-up tilt test, vasovagal syndrome, syncope, menstrual cycle, menopause
\end{abstract}

\section{Introduction}

A woman's body is subject to continuous but changing influence from the endocrine system throughout her life. After puberty, estrogen and progesterone levels rise and fall alternately according to the phase of the menstrual cycle. Later, during the perimenopausal period, estrogen and progesterone levels begin to fall with synchronous increase in gonadotropin (FSH and LH) levels [1]. Numerous studies have confirmed that female hormones influence the cardiovascular system. Estrogen receptors are present on the endothe- lial cells and vascular smooth muscles, and are involved in the regulation of vascular resistance [2-4]. Female hormones also influence the sensitivity of adrenergic receptors in the sympathetic nervous system, as well as plasma volume and skin blood flow [5-7]. Moreover, they may influence endothelial function, leading to abnormal vasodilation of peripheral arteries during vasovagal syncope in response to orthostatic stress.

Given the role female sex hormones play in the cardiovascular system, it is plausible that they also modulate the reflex responses which are responsible for the occurrence of vasovagal syncope. The

Address for correspondence: Dr. Joanna Jędrzejczyk-Spaho, Department of Coronary Artery Disease and Heart Failure, Institute of Cardiology, Medical School of Jagiellonian University, John Paul II Hospital, ul. Prądnicka 80, 31-202 Kraków, Poland, tel: +48 1261422 18,fax: +48 1243343 76, e-mail: joanna_spaho@interia.pl

Received: 27.05.2016 Accepted: 05.08.2016 
mechanism of reflex syncope is based on the fact that orthostatic stress, emotional stimuli and pain can induce transient dysfunction of the autonomic nervous system, leading to a drop in blood pressure, bradycardia and finally, loss of consciousness. Paradoxically, peripheral vasodilation caused by endothelial dysfunction may also play a key role in inappropriate, excessive hypotension during orthostatic stress in individuals with vasovagal syndrome (VVS) [8].

The incidence of vasovagal syncope is higher in women than in men $[9,10]$. There are some publications revealing that results of the head-up tilt test (HUTT) are not influenced by menstrual cycle phase [11]. The HUTT has been used to diagnose reflex syncope for 30 years, but today, in the light of the results of the ISSUE-3 placebo-controlled study, practice has changed and is directed in favor of risk stratification than diagnosis. A positive HUTT indicates susceptibility to hypotension and vasodepression; it may also indicate that a patient will benefit less from pacing therapy, because of a higher risk of syncope recurrence [12].

The purpose of this study was to evaluate the prevalence of syncope induced by HUTT and the type of positive vasovagal response to the orthostatic stress in a relationship to the estimated phase of menstrual cycle (follicular phase, luteal phase) or menopause.

\section{Methods}

Participants were recruited consecutively from a population of 1,275 patients with recurrent syncopal events. The sample consisted of 500 women aged $13-89$ years (median age 37.5 years, interquartile range [IQR] 23-59) with the history of faints with probable vasovagal etiology, who had been referred for to the Syncope Unit of Department of Coronary Disease and Heart Failure at the Jagiellonian University Medical College, John Paul II Hospital in Krakow for further investigation. Patients with underlying cardiovascular disease, which might cause episodes of syncope, and patients with symptomatic orthostatic hypotension due to cardiovascular or neurological diseases were excluded from the study.

Informed consent was obtained from all individual participants included in the study. The study was conducted in accordance with the Declaration of Helsinki and approved by the Bioethical Committee at Jagiellonian University in Krakow.

After an initial assessment, all women underwent the HUTT, which was administered accord- ing to the guidelines of the European Society of Cardiology [13]. The initial evaluation included medical history, clinical examination, measurement of blood pressure (supine and standing) and electrocardiogram [13].

The HUTT was administered in a silent, slightly darkened room maintained at a constant temperature. Interfering external stimuli (acoustic, visual and other) were excluded during the test. The test was administered according to one of two randomly selected protocols: the Westminster protocol (passive phase of 60-degree tilt lasting for $45 \mathrm{~min}$ ) or Italian protocol (passive tilting phase of 60 -degree tilt lasting for $20 \mathrm{~min}$ ). Where the passive phase produced a negative results it was fallowed by sublingual administration of $0.4 \mathrm{mg}$ nitroglycerine (NTG)-provocation phase - which lasted for another $20 \mathrm{~min}$ in Westminster protocol and for another $15 \mathrm{~min}$ in Italian protocol. The HUTT continued until syncope occurred or until the protocol was completed [13]. During the HUTT, an electrocardiogram (ECG) recording was made and beat-to-beat blood pressure was monitored continuously using a validated, non-invasive method (Nexfin, BMEYE, The Netherlands).

The result of the HUTT was classified as positive if the test induced reflex hypotonia and/or bradycardia with concomitant syncope, similar to spontaneous syncope. Intensive prodromal symptoms without syncope provocation were classified as a doubtful test result [12]. The VASIS (The Vasovagal Syncope International Study) classification was used to classify vasovagal responses to tilt testing, based on heart rhythm and blood pressure pattern induced by orthostatic stress. Based on a modification of the original VASIS classification we distinguished three types of vasovagal response:

- Mixed: heart rate falls at the time of syncope but the ventricular rate does not fall as low as $40 \mathrm{bpm}$, or falls as low as $40 \mathrm{bpm}$ for less than $10 \mathrm{~s}$;

- Cardioinhibition: heart rate falls to a ventricular rate of $40 \mathrm{bpm}$ or lower for more than $10 \mathrm{~s}$ and/or there is an asystole lasting more than $3 \mathrm{~s}$;

- Vasodepression: heart rate does not fall more than $10 \%$ from its peak at the time of syncope. For the purposes of our study the menstrual cycle phase was determined on the basis of the patient's usual length of menstrual cycle and the day of the cycle on which she was tested, assuming constant length of the luteal phase (14 days). Patients reporting irregularity regarding the length of their menstrual cycle have been excluded from the study. Normal cycle length is considered from 21 to 
Table 1. Distribution of protocols used for the head-up tilt test, $p=$ NS.

\begin{tabular}{|c|c|c|c|c|c|c|c|c|}
\hline \multirow[t]{3}{*}{ Tilt test protocol } & \multirow{2}{*}{\multicolumn{2}{|c|}{ All women }} & \multirow{2}{*}{\multicolumn{2}{|c|}{$\begin{array}{l}\text { Post-menopausal } \\
\text { women }\end{array}$}} & \multicolumn{4}{|c|}{ Menstruating women } \\
\hline & & & & & \multicolumn{2}{|c|}{ Follicular phase } & \multicolumn{2}{|c|}{ Luteal phase } \\
\hline & $\mathbf{N}$ & $\%$ & $\mathbf{N}$ & $\%$ & $\mathbf{N}$ & $\%$ & $\mathbf{N}$ & $\%$ \\
\hline Westminster protocol & 116 & 23.2 & 38 & 20.5 & 45 & 26.5 & 33 & 22.8 \\
\hline $\begin{array}{l}\text { Westminster protocol with } \\
\text { pharmacological provocation }\end{array}$ & 79 & 15.8 & 16 & 8.6 & 35 & 20.6 & 28 & 19.3 \\
\hline Italian protocol & 305 & 61.0 & 131 & 70.8 & 90 & 52.9 & 84 & 57.9 \\
\hline Passive standing alone & 116 & 23.2 & 40 & 21.6 & 45 & 26.5 & 31 & 21.4 \\
\hline $\begin{array}{l}\text { Pharmacological provocation } \\
\text { with nitroglycerine }\end{array}$ & 384 & 78.6 & 145 & 78.4 & 125 & 73.5 & 114 & 78.6 \\
\hline
\end{tabular}

35 days and it is an individual feature. The ovarian cycle consists of the follicular phase, ovulation, and luteal phase. The luteal phase is defined as the interval following but not including the luteinizing hormone peak (it is $24 \mathrm{~h}$ before ovulation) up to the day before menstruation. Small part of the female population (about 5\%) has a short luteal phase defined as 12 days or less but the majority has a normal luteal phase length, which is $14.13 \pm 1.41$ days [14]. We used the below formula to estimate the day of the menstrual cycle:

[usual length of the menstrual cycle] -14 days $=\mathrm{X}$

The day of the cycle at the moment of the HUTT $\leq \mathrm{X}$ - follicular phase

The day of the cycle at the moment of the HUTT $>\mathrm{X}$ - luteal phase

Although the normal length of the menstrual cycle is variable for different women (21-35 days), the constant length of luteal phase allows us to estimate with high probability the phase of menstrual cycle for women with regular length of menstrual cycle.

\section{Ethical approval and informed consent}

All procedures performed in studies involving human participants were in accordance with the ethical standards of the institutional and/or National Research Committee and with the 1964 Helsinki declaration with its later amendments, or comparable ethical standards.

Informed consent was obtained from all individual participants included in the study.

\section{Statistical analysis}

In order to assess the normal character of variables, the Kolmogorov-Smirnov test and ShapiroWilk test were used. The quantitative variables were not normally distributed and are presented as medians with the IQR. Categorical variables are presented as percentages or frequency distributions. Data were analyzed using the Mann-Whitney $\mathrm{U}$ test. Categorical variables were analyzed using the $\chi^{2}$ test. The significance threshold was set at $\mathrm{p}<0.05$. The analysis was conducted using STATISTICA software (Stat-Soft) version 10.0.

\section{Results}

Out of the 500 women enrolled in the study, $185(37.0 \%)$ were in menopause, $170(34.0 \%)$ were in the follicular phase when tested, and 145 (29.0\%) were in the luteal phase (NS).

The Westminster version of the passive tilt test was administered to $116(23.2 \%)$ patients and further 79 (15.8\%) patients received the Westminster passive protocol complemented by pharmacological provocation with NTG; the Italian version of the HUTT was administered to 305 $(61.0 \%)$ women. In total, NTG (provoking agent) was administered to $384(78.6 \%)$ patients. There was no difference in either the distribution of test protocols or the frequency of use of pharmacological provocation according to menopausal status or menstrual phase (Table 1).

The tilt test was negative in $75(15.0 \%)$ patients, doubtful in $45(9.0 \%)$ and positive in 380 $(76.0 \%)$ patients. In patients with a positive HUTT, the distribution of vasovagal responses was as follows: mixed: $43.8 \%$; cardioinhibitory: $17.0 \%$ 
Table 2. Results of head-up tilt test (HUTT) in relation to the estimated menstrual phase and menopausal status.

\begin{tabular}{|c|c|c|c|c|c|c|c|c|c|c|}
\hline \multirow[t]{3}{*}{ HUTT } & \multirow{2}{*}{\multicolumn{2}{|c|}{ All women }} & \multirow{2}{*}{\multicolumn{2}{|c|}{$\begin{array}{l}\text { Menopausal } \\
\text { women }\end{array}$}} & \multirow{2}{*}{\multicolumn{2}{|c|}{$\begin{array}{c}\text { Menstruating } \\
\text { women }\end{array}$}} & \multicolumn{4}{|c|}{ Menstruating women } \\
\hline & & & & & & & \multicolumn{2}{|c|}{ Follicular phase } & \multicolumn{2}{|c|}{ Luteal phase } \\
\hline & $\mathbf{N}$ & $\%$ & $\mathbf{N}$ & $\%$ & $\mathbf{N}$ & $\%$ & $\mathbf{N}$ & $\%$ & $\mathbf{N}$ & $\%$ \\
\hline Negative result & 75 & 15.0 & 25 & 13.5 & 50 & 15.9 & 33 & 19.4 & 17 & 11.7 \\
\hline Positive result & 380 & 76.0 & 141 & 76.2 & 239 & 75.9 & 123 & 72.4 & 116 & 80.0 \\
\hline Doubtful result & 45 & 9.0 & 19 & 10.3 & 26 & 8.3 & 14 & 8.2 & 12 & 8.3 \\
\hline Mixed response & 219 & 43.8 & 65 & $35.1 *$ & 154 & $48.8^{*}$ & 72 & 42.4 & 82 & 56.6 \\
\hline $\begin{array}{l}\text { Cardioinhibitory } \\
\text { response }\end{array}$ & 85 & 17.0 & 23 & $12.4^{*}$ & 62 & $19.7^{*}$ & 34 & 20.0 & 28 & 19.3 \\
\hline Without asystole & 10 & 2.0 & 4 & 2.2 & 6 & 1.9 & 4 & 2.4 & 2 & 1.4 \\
\hline With asystole & 75 & 15.0 & 19 & 10.3 & 56 & 17.8 & 30 & 17.6 & 26 & 17.9 \\
\hline $\begin{array}{l}\text { Vasodepressive } \\
\text { response }\end{array}$ & 76 & 15.2 & 53 & $28.6^{*}$ & 23 & $7.3^{*}$ & 17 & $10.0^{*}$ & 6 & $4.1^{*}$ \\
\hline
\end{tabular}

${ }^{*} p<0.00001$

(cardioinhibitory with asystole: $15.0 \%$ ); vasodepressive: $15.6 \%$. Mixed and cardioinhibitory responses were more frequent in pre-menopausal patients than post-menopausal patients $(48.8 \%$ vs. $35.1 \%$ and $19.7 \%$ vs. $12.4 \%$, respectively; both $\mathrm{p}<0.00001$ ), whereas vasodepressive responses were less frequent in pre-menopausal women (7.3\% vs. $28.6 \%$; $p<0.00001)$. Vasodepressive responses were more frequent in women in follicular phase than in women in the luteal phase $(10.0 \%$ vs. $4.1 \%$, respectively, $\mathrm{p}<0.00001$ ) (Table 2 ).

Average duration of the passive phase of the HUTT, duration of the pharmacological provocation phase, and the total test duration were similar in pre-menopausal and post-menopausal women, as well as in women in the follicular and luteal phases of menstrual cycle.

Mean age of the first syncope was lower in menstruating women (median: 15 years, IQR: 14-56) than in post-menopausal women (median: 30 years, IQR: $10-21 ; \mathrm{p}<0.0001)$.

\section{Discussion}

The main result of our study is that the prevalence of vasovagal syncope was similar in menstruating and postmenopausal women. The important finding is that the type of vasovagal response to the orthostatic stress induced by the HUTT varied according to the estimated menstrual phase and menopausal status.

In our study, vasodepressive VVS was more frequent in post-menopausal women, whereas in menstruating women cardioinhibitory and mixed responses were more common. One possible explanation for this observation might be the postulated difference in the mechanism of vasovagal syncope in elderly patients. In older patients, vasovagal syncope is sometimes called vasovagal disease. Most investigations into postulated pathomechanisms of vasovagal syncope, such as reductions in sympathetic nerve activity [15], myocardial contractility [16], circulating noradrenaline, renin and endothelin c [17] have been carried out in younger samples (mean age of 42.5 years) and the results cannot be easily generalized for the whole population. The humoral response to orthostatic stress changes with age; the change is probably related to alterations in the autonomic nervous system. It is probable that in older people, the main mechanism for maintaining blood pressure during orthostasis is the sympathetic mechanism, given that plasma renin activity declines with age [18]. Another difference is that in the classic, isolated VVS, blood pressure and heart rate are constant until sudden drop immediately before onset of syncope, whereas in older patients there is a more gradual decrease in blood pressure [19].

As well as the difference in pathomechanism, the clinical pattern of VVS is also different in older patients. There is evidence that early onset VVS is different from the later onset forms, which are often associated with autonomic disturbances, such as carotid sinus hypersensitivity, post-prandial hypotension and other symptoms of autonomic dysfunction [20]. In this study, the mean age of first 
syncope was lower in menstruating patients than in post-menopausal patients, which may reflect a difference in pathology, probably related to the autonomic nervous system. The observed difference in the prevalence of vasodepressive vasovagal responses in post-menopausal women is also consistent with the majority of available evidence. Studies carried by Kochiadakis et al. [21] and Kurbaan et al. [22] show that younger people are more likely to show bradycardia in response to orthostatic stress than hypotension. Another study carried out on elderly population ( $>65$ years old) proves that in this group of subjects, the vasodepressor form of carotid sinus hypersensitivity occurs more often [23]. In an article on differential diagnosis of isolated vasovagal syndrome and vasovagal disease, Alboni et al. [20] stated that in spontaneous syncope, the cardioinhibitory and vasodepressive forms are similarly frequent.

Our finding has important therapeutic implications. The prognosis for reflex syncope is worse in older people due to the clinical features: prodromal symptoms of vasovagal syncope are often absent or of very short duration and therefore, injuries due to fainting are more likely, as is amnesia following the loss of consciousness, which may result in a misdiagnosis of unexplained falling rather than fainting $[19,24]$. Thus, a more invasive therapy, including pacemaker implantation, is often considered in elderly patients, according to the European Society of Cardiology recommendations (IIB class of recommendation for HUTT-induced syncope) [13]. The guidelines emphasize, however, that pacing is efficacious only for asystolic reflex and has no role in combating hypotension. This explains why older patients with vasodepressive VVS do not benefit from this kind of therapy.

Our study also revealed that the vasovagal mechanism differs according to the estimated phase of the menstrual cycle. We observed that in menstruating women in whom we have calculated the probable phase of menstrual cycle, the vasodepressive VVS was more frequent in the follicular than in the luteal phase (10\% vs. $4 \%$, respectively, $\mathrm{p}<0.00001)$. We may try to explain this observation by the higher estrogen serum concentration in the follicular than in the luteal phase. Estrogens are responsible for increased production of nitric oxide, a potent vasodilator which may increase susceptibility to vasodepressive vasovagal syncope. There are data showing that the endothelial hyperactivity due to upregulation of nitric oxide synthase is essential for regulation of vascular tone in VVS, and may lead to hypotension exceed- ing the capacity of compensatory mechanisms $[3,4,8]$. Additionally, an increased vasoconstriction, due to the noradrenaline function observed in the luteal phase, may inhibit vasodilatation provoked by orthostatic stress [25].

The difference of vasovagal mechanism regarding different phases of menstrual cycle is surprising, taking into account that most research indicates that although female sex hormones influence regulation of the sympathetic nervous system and cardiovascular system, heart rate, blood pressure and orthostatic stress tolerance during the tilt test do not vary with menstrual phase [26, 27]. Meendering et al. [26] demonstrated that during the luteal phase of the menstrual cycle, there is a greater increase blood flow in the skin in response to elevated ambient temperature than in the follicular phase. From a theoretical perspective, one might expect this to reduce orthostatic stress tolerance, however no variations in orthostatic stress tolerance were observed during the course of the menstruation cycle [26].

Previous research indicates that total peripheral resistance does not vary with hormone levels, despite the well-documented influence of estrogen and progesterone on sympathetic nervous activity [5, 28]. The evidence on changes in baroreflex sensitivity over the course of the menstrual cycle is more equivocal. The study by Meendering et al. [26], which was cited above, found that the acceleration of heart rate in response to passive tilting did not vary according to phase of the menstrual cycle, which the authors interpreted as a lack of difference in baroreflex sensitivity, however another study reported that baroreflex sensitivity is greater in the follicular phase of the menstrual cycle [18]. When interpreting these results, it should be remembered that female hormones have multiple functions and that different paths of action may counteract each other. For example, estrogen which has a vasodilatatory effect through its effect on nitric oxide levels [29], may also cause an increase in blood plasma volume [30], thus having a bidirectional effect on the blood pressure.

Another study which investigated how menstrual cycle phase influenced the course of the HUTT found rather different results from ours. Zyśko et al. [11] have examined a group of 183 premenopausal women. They divided menstrual cycle into four phases based on the self-report menstrual status: perimenstrual $(\mathrm{M})$, preovulatory $(\mathrm{F})$, periovulatory $(\mathrm{O})$ and postovulatory $(\mathrm{L})$. They observed that cardioinhibitory reactions were 
less frequent in the post-ovulatory phase than in the perimenopausal and ovulatory phases [11]. However, it is difficult to make direct comparison between the studies owing to differences in how the menstrual cycle was divided.

\section{Limitations of the study}

The observational nature of this study constitutes a limitation. We did not compare groups on patient characteristics other than menstrual status and it is possible that there are explanations for the observed differences between groups other than menstrual status.

Another limitation of our study is the lack of analysis of sex hormone levels on the day of the HUTT. Our data on menstrual phase was based on patients' self-reports of standard cycle length and position in their cycle; we assumed a constant luteal phase. On the other hand, our results are based on a fairly large sample (301 pre-menopausal women), whichmakes us believe that our description of the influence of female sex hormones on the outcome of the HUTT is accurate.

\section{Conclusions}

1. Among women referred for HUTT, the prevalence of the VVS did not differ between those that were pre and post menopausal.

2. The incidence of vasodepressive reactions is higher in post-menopausal women than in premenopausal women.

3. In our sample, age of first syncope presentation was lower in menstruating women than in post-menopausal women, which may reflect a difference in the pathomechanism of vasovagal syncope (classical VVS or vasovagal disease).

4. In pre-menopausal women, vasodepressive VVS is more frequent in the estimated follicular phase than in the luteal phase.

\section{Acknowledgements}

The study was supported by a grant from the Polish National Science Center, N402 475040.

\section{Conflict of interest: None declared}

\section{References}

1. Fritz MA, Speroff L. Clinical Gynecologic endocrinology and infertility. In: Speroff L ed. Menstrual Cycle. 5th Edn. Williams \& Wilkins, Lippincott, 2010; 210-263.

2. Tostes RC, Nigro D, Fortes ZB, et al. Effects of estrogen on the vascular system. Braz J Med Biol Res. 2003; 36(9): 1143-1158, doi: 10.1590/s0100-879x2003000900002, indexed in Pubmed: 12937779.

3. Sudhir K, Jennings GL, Funder JW, et al. Estrogen enhances basal nitric oxide release in the forearm vasculature in perimenopausal women. Hypertension. 1996; 28(3): 330-334, doi: 10.1161/01. hyp.28.3.330, indexed in Pubmed: 8794812.

4. Rosselli M, Imthurm B, Macas E, et al. Circulating nitrite/nitrate levels increase with follicular development: indirect evidence for estradiol mediated NO release. Biochem Biophys Res Commun. 1994; 202(3): 1543-1552, doi: 10.1006/bbrc.1994.2107, indexed in Pubmed: 8060338.

5. Freedman RR, Girgis R. Effects of menstrual cycle and race on peripheral vascular alpha-adrenergic responsiveness. Hypertension. 2000; 35(3): 795-799, doi: 10.1161/01.hyp.35.3.795, indexed in Pubmed: 10720597.

6. Oian P, Tollan A, Fadnes HO, et al. Transcapillary fluid dynamics during the menstrual cycle. Am J Obstet Gynecol. 1987; 156(4): 952-955, indexed in Pubmed: 3578405.

7. Hessemer V, Brück K. Influence of menstrual cycle on shivering, skin blood flow, and sweating responses measured at night. J Appl Physiol (1985). 1985; 59(6): 1902-1910, indexed in Pubmed: 4077797.

8. Pietrucha AZ. Endothelial function in vasovagal syncope. Expert Rev Cardiovasc Ther. 2014; 12(12): 1387-1389, doi: 10.1586/14 779072.2014.982095, indexed in Pubmed: 25410020.

9. Park J, Jang SYi, Yim HR, et al. Gender difference in patients with recurrent neurally mediated syncope. Yonsei Med J. 2010; 51(4): 499-503, doi:10.3349/ymj.2010.51.4.499, indexed in Pubmed: 20499413.

10. Convertino VA. Gender differences in autonomic functions associated with blood pressure regulation. Am J Physiol. 1998; 275(6 Pt 2): R1909-R1920, indexed in Pubmed: 9843880.

11. Zyśko D, Gajek J, Terpiłowski L, et al. Effects of the menstrual cycle phases on the tilt testing results in vasovagal patients. Arch Gynecol Obstet. 2012; 286(2): 429-435, doi: 10.1007/s00404012-2308-4, indexed in Pubmed: 22491807.

12. Sutton R, Brignole M. Twenty-eight years of research permit reinterpretation of tilt-testing: hypotensive susceptibility rather than diagnosis. Eur Heart J. 2014; 35(33): 2211-2212, doi: 10.1093/eurheartj/ehu255, indexed in Pubmed: 24980488.

13. Moya A, Sutton R, Ammirati F, et al. Task Force for the Diagnosis and Management of Syncope, European Society of Cardiology (ESC), European Heart Rhythm Association (EHRA), Heart Failure Association (HFA), Heart Rhythm Society (HRS). Guidelines for the diagnosis and management of syncope (version 2009). Eur Heart J. 2009; 30(21): 2631-2671, doi: 10.1093/eurheartj/ ehp298, indexed in Pubmed: 19713422.

14. Lenton EA, Landgren BM, Sexton L. Normal variation in the length of the luteal phase of the menstrual cycle: identification of the short luteal phase. Br J Obstet Gynaecol. 1984; 91(7): 685-689, indexed in Pubmed: 6743610.

15. Morillo CA, Eckberg DL, Ellenbogen KA, et al. Vagal and sympathetic mechanisms in patients with orthostatic vasovagal syncope. Circulation. 1997; 96(8): 2509-2513, doi: 10.1161/01. cir.96.8.2509, indexed in Pubmed: 9355886.

16. Mizumaki K, Fujiki A, Tani M, et al. Left ventricular dimensions and autonomic balance during head-up tilt differ between patients with isoproterenol-dependent and isoproterenol-independent neurally mediated syncope. J Am Coll Cardiol. 1995; 26(1): 164-173, doi: 10.1016/0735-1097(95)00120-o, indexed in Pubmed: 7797746. 
17. Ellenbogen KA, Morillo CA, Wood MA, et al. Neural monitoring of vasovagal syncope. Pacing Clin Electrophysiol. 1997; 20(3 Pt 2): 788-794, doi:10.1111/j.1540-8159.1997.tb03905.x, indexed in Pubmed: 9080511.

18. Kenny RA, Lyon CC, Bayliss J, et al. Reduced plasma renin activity in elderly subjects in response to vasovagal hypotension and head-up tilt. Age Ageing. 1987; 16(3): 171-177, doi: 10.1093/ ageing/16.3.171, indexed in Pubmed: 3300207.

19. Brignole M, Menozzi C, Del Rosso A, et al. New classification of haemodynamics of vasovagal syncope: beyond the VASIS classification. Analysis of the pre-syncopal phase of the tilt test without and with nitroglycerin challenge. Vasovagal Syncope International Study. Europace. 2000; 2(1): 66-76, doi: 10.1053/ eupc.1999.0064, indexed in Pubmed: 11225598.

20. Alboni P, Brignole M, Ettore C. Degli Uberti. Is vasovagal syncope a disease? Hellenic J Cardiol. 2008; 49: 61-64.

21. Kochiadakis GE, Papadimitriou EA, Marketou ME, et al. Autonomic nervous system changes in vasovagal syncope: is there any difference between young and older patients? Pacing Clin Electrophysiol. 2004; 27(10): 1371-1377, doi: 10.1111/j.1540-8159.2004.00641.x, indexed in Pubmed: 15511246.

22. Kurbaan AS, Bowker TJ, Wijesekera N, et al. Age and hemodynamic responses to tilt testing in those with syncope of unknown origin. J Am Coll Cardiol. 2003; 41(6): 1004-1007, doi: 10.1016/ s0735-1097(02)02967-4, indexed in Pubmed: 12651049.

23. McIntosh SJ, Lawson J, Kenny RA. Clinical characteristics of vasodepressor, cardioinhibitory, and mixed carotid sinus syndrome in the elderly. Am J Med. 1993; 95(2): 203-208, doi: 10.1016/00029343(93)90261-m, indexed in Pubmed: 8356984.
24. O'Dwyer C, Bennett K, Langan Y, et al. Amnesia for loss of consciousness is common in vasovagal syncope. Europace. 2011; 13(7): 1040-1045, doi:10.1093/europace/eur069, indexed in Pubmed: 21436135.

25. Goldstein DS, Levinson P, Keiser HR. Plasma and urinary catecholamines during the human ovulatory cycle. Am J Obstet Gynecol. 1983; 146(7): 824-829, indexed in Pubmed: 6869453.

26. Meendering JR, Torgrimson BN, Houghton BL, et al. Menstrual cycle and sex affect hemodynamic responses to combined orthostatic and heat stress. Am J Physiol Heart Circ Physiol. 2005; 289(2): H631-H642, doi: 10.1152/ajpheart.00029.2005, indexed in Pubmed: 15778279.

27. Claydon VE, Younis NR, Hainsworth R. Phase of the menstrual cycle does not affect orthostatic tolerance in healthy women. Clin Auton Res. 2006; 16(2): 98-104, doi: 10.1007/s10286-0060330-y, indexed in Pubmed: 16683068.

28. Minson CT, Halliwill JR, Young TM, et al. Influence of the menstrual cycle on sympathetic activity, baroreflex sensitivity, and vascular transduction in young women. Circulation. 2000; 101(8): 862-868, indexed in Pubmed: 10694525.

29. Sader MA, Celermajer DS. Endothelial function, vascular reactivity and gender differences in the cardiovascular system. Cardiovasc Res. 2002; 53(3): 597-604, doi: 10.1016/s00086363(01)00473-4, indexed in Pubmed: 11861030.

30. Stachenfeld NS, Taylor HS, Leone CA, et al. Oestrogen effects on urine concentrating response in young women. J Physiol. 2003; 552(Pt 3): 869-880, doi: 10.1113/jphysiol.2003.046920, indexed in Pubmed: 12923212. 